\title{
Cosmic ray particles from exploding massive stars with winds
}

\author{
P. L. Biermann $1,2,3,4$ \\ ${ }^{1}$ Max Planck Institute for Radio Astronomy Bonn (MPIfR), Bonn, Germany \\ ${ }^{2}$ Department of Physics \& Astronomy, University of Alabama, Tuscaloosa, AL, USA \\ ${ }^{3}$ Institute of Nuclear Physics (IKP), KIT, Karlsruhe, Germany \\ ${ }^{4}$ Department of Physics \& Astronomy, University of Bonn, Bonn, Germany
}

Correspondence to: P. L. Biermann (plbiermann@mpifr-bonn.mpg.de)

Received: 25 March 2014 - Revised: 12 May 2014 - Accepted: 13 May 2014 - Published: 27 June 2014

\begin{abstract}
The origin of cosmic rays is still unsettled. Many sources have been proposed over the years, and exploding stars still provide the most promising candidates. Here we examine one of these scenarios, and compare the resulting predictions with data: Massive stars have winds, and when these stars explode, the resulting shock runs through the wind. The observable phenomenon is called radio-supernova, and many have been observed in non-thermal radio emission. This emission allows to determine the magnetic field in the wind as a function of radius, and so allows to check, whether such explosions can achieve the high energies required and also explain the flux and the spectra of cosmic rays. The observations show this to be the case, and so we conclude that radio supernovae can explain the high-energy Galactic cosmic rays over the entire energy range, and that the spectral predictions are compatible with observations.
\end{abstract}

\section{Introduction}

Cosmic rays have now been observed over a large range of particle energies, exceeding by far the energies achievable on Earth, such as at the LHC. However, since the rate of high energy cosmic ray particles is low, it would be helpful to fully understand where they come from, so that we can perhaps utilize the sites of origin, acceleration, and possibly interaction to perform tests of what the physics are of particles at such extreme energies. In almost all scenarios diffusive shock acceleration is used (e.g. Drury, 1983; Berezinsky et al., 1990).

Classical supernova remnants (Baade and Zwicky, 1934), that is stars exploding into the interstellar medium, do not provide the circumstances required to accelerate particles to such high energies (Lagage and Cesarsky, 1983) if the interstellar observed magnetic field is used only. However, if the shock itself can enhance the magnetic field to some reasonable fraction of the ram pressure (Bell and Lucek, 2001), then much higher energies can be achieved. Also, pulsars often have a powerful magnetic wind, again resulting in particle acceleration (de Jager, 2009; Tibolla et al., 2013), quite pos- sibly to very high energies. Similarly, other manifestations of massive star explosions, such as Gamma Ray Bursts (e.g. Dermer, 2002), or high-mass X-ray binary stars, or micro quasars (e.g. Heinz and Sunyaev, 2002), can induce efficient particle acceleration. Very massive stars are often found in a super bubble of hot gas, and when these stars explode into this environment, then that shock expands into this tenuous, hot gas. That shock can accelerate particles to high energies as well. Finally, as massive stars explode into their own wind, that shock runs through a medium which is already magnetic and hot, and due to the radial density gradient of the wind, does not slow down immediately. Such a shock also accelerates particles, in this case, with the elemental abundances of the wind. Of all these scenarios, the last one can now be examined using new observations, namely radio observations of supernovae. These radio observations allow a determination of the magnetic field in the wind of the stars, and its dependence on the radius. This then allows a definitive statement on the maximal energy to which particles can be accelerated at these specific sites. 


\section{Radiosupernovae and energetic particles}

What physical conditions at the acceleration regions do the cosmic ray data indicate? Galactic cosmic rays are typically invoked to explain nuclei up to energies of about $3 \times 10^{18} \mathrm{eV}$, where there is a transition to a different, again flatter spectrum. There is also a limit on confinement of the particles in the Galaxy and a change in chemical composition. Very often, when a supernova is detected, radio telescopes immediately detect emission that is strongly time-dependent, and clearly produced by energetic particles in the shock running through the stellar wind. There are now a number of such events observed, which allow a determination of the magnetic field with a typical value of $B=1 \mathrm{Gau} ß$ at a radial distance of $r=10^{16} \mathrm{~cm}$ (e.g. Fransson and Björnsson, 1998; Soderberg et al., 2005, 2006, 2010; Krauss et al., 2011; Milisavljevic et al., 2013). These magnetic fields are much higher than what direct observations of early type stars suggest (e.g. Bieging et al., 1989). Therefore there are two possible explanations: (a) the star strongly increases its magnetic field in the wind just before the explosion. This may be plausible if indeed the explosion is connected to magnetic fields, as suggested by Bisnovatyi-Kogan (1970), and worked out in much more detail by Bisnovatyi-Kogan and Moiseenko (2008). (b) Another option is that the magnetic fields are strongly enhanced in the shock-wave itself through violent instabilities, as proposed by Bell and Lucek (2001). For our query we do not need to differentiate between these two scenarios, because in both alternatives the magnetic field is proportional to $r^{-1}$. As a consequence the product of magnetic field and radial distance is a constant. The shock-wave in a wind compresses the wind material into a layer of about $r / 4$ for an adiabatic gas index of 5/3 and assuming a very strong shock. Since the observations suggest that the shock speed often approaches $1 / 10$ of the speed of light, a strong shock is assured. This implies that the maximum energy with which particles can be contained in the acceleration region is given by $E_{\max }=Z e B r / 8$, where $Z e$ is the charge of the nucleus considered, and $B$ is determined at that radius $r$, since the shocked region needs to contain the diameter of the Larmor motion. For the numbers given above this leads to $\sim 5 \times 10^{17} \mathrm{ZeV}$ as the maximally attainable particle energy. Thus the ankle energy of about $3 \times 10^{18} \mathrm{eV}$ can readily be reached for for nuclei with $Z>2$. Using the particle scattering model proposed by Jokipii (1987) it can easily be shown that the very high energies derived above can be reached in the time available.

\section{Spectra and abundances}

The cosmic ray spectrum depends on adiabatic losses, injection history, geometry, and electric fields, which always occur in a situation with motion transverse to the magnetic field; this results in electric field drifts further accelerating the particles. In a highly chaotic situation driven by plasma instabilities there are always strong magnetic field components transverse to the shock normal. Only for wind-supernovae a rather specific prediction has been made (Biermann, 1993), using maximal turbulence in the shock region, resulting in a spectrum scaling as $E^{-7 / 3}$, implying a predicted observed spectrum of $E^{-8 / 3}$ using Kolmogorov transport (Kolmogorov, 1941). We emphasize that this prediction does not depend on whether the magnetic fields derive from enhancement via the mechanism of Bell and Lucek (2001), or simply arise from the wind. Beyond the knee energy of about $10^{15} \mathrm{eV}$, the acceleration efficiency is reduced by the decreasing of the drift energy gain, so for these energies a spectrum of about $E^{-3.1}$ has been predicted (Biermann, 1993). Taking into account the error bars both in the prediction and in the data, as seen today, these predictions are consistent with observations.

\section{Conclusions}

We have shown that observations of wind-supernovae unequivocally demonstrate that these objects are capable of accelerating cosmic rays up to energies of a few $10^{18} \mathrm{eV}$. Consequently, of the many different proposed acceleration sites to account for Galactic cosmic rays, wind-supernovae are no longer only a hypothetical source. For this specific source type also the spectra and abundances can be explained. Other sources are not ruled out yet, and more detailed observations are required to differentiate, such as molecular emission lines given by level populations dominated by low energy cosmic rays (see, e.g. Becker et al., 2011).

Acknowledgements. Extensive discussion with many colleagues are acknowledged, especially with Julia Becker Tjus, Andreas Brunthaler, Rolf Chini, Ralph Engel, Nayantara Gupta, Athina Meli, Biman B. Nath, Eun-Suk Seo, Vitor de Souza, Todor Stanev, and Carlos J. Todero Peixoto.

The service charges for this open access publication have been covered by the Max Planck Society.

Edited by: J. Tjus

Reviewed by: T. Stanev and one anonymous referee

\section{References}

Baade, W. and Zwicky, F.: Cosmic rays from supernovae, P. Natl. Acad. Sci. USA, 20, 259-263, 1934.

Becker, J. K., Black, J. H., Safarzadeh, M., and and Schuppan, F.: Tracing the Sources of Cosmic Rays with Molecular Ions, Astrophys. J. Lett., 739, L43, doi:10.1088/2041-8205/739/2/L43, 2011.

Bell, A. R. and Lucek, S. G.: Cosmic ray acceleration to very high energy through the non-linear amplification by cosmic rays of the seed magnetic field, Mon. Not. R. Astron. Soc., 321, 433438, doi:10.1046/j.1365-8711.2001.04063.x, 2001. 
Berezinsky, V. S., Bulanov, S. V., Dogiel, V. A., Ginzburg, V. L., and Ptuskin, V. S.: Astrophysics of Cosmic Rays, Amsterdam, North-Holland, 534 pp., 1990.

Bieging, J. H., Abbott, D. C., and Churchwell, E. B.: A survey of radio emission from Galactic OB stars, Astrophys. J., 340, 518536, 1989.

Biermann, P. L.: Cosmic rays. 1. The cosmic ray spectrum between $10^{4} \mathrm{GeV}$ and $3 \times 10^{9} \mathrm{GeV}$, Astron. Astrophys., 271, 649-661, 1993.

Bisnovatyi-Kogan, G.: The Explosion of a Rotating Star As a Supernova Mechanism, Astron. Zh., 47, 813-816, 1970 (translation: Sov. Astron. A. J., 14, 652-655, 1970).

Bisnovatyi-Kogan, G. S. and Moiseenko, S. G.: Magnetorotational Supernovae with Jets, Chinese J. Astron. Ast., Supplement, 8, 330-340, 2008.

de Jager, O.: Unidentified Gamma-Ray Sources as Ancient Pulsar Wind Nebulae, ICRC 2009, 1-4, arXiv:0906.2644, 2009.

Dermer, C. D.: Neutrino, Neutron, and Cosmic-Ray Production in the External Shock Model of Gamma-Ray Bursts, Astrophys. J., 574, 65-87, 2002.

Drury, L. O'C.: An introduction to the theory of diffusive shock acceleration of energetic particles in tenuous plasmas, Rep. Prog. Phys., 46, 973-1027, doi:10.1088/0034-4885/46/8/002, 1983.

Fransson, C. and Björnsson, C.-I.: Radio Emission and Particle Acceleration in SN 1993J, Astrophys. J., 509, 861-878, doi:10.1086/306531, 1998.

Heinz, S. and Sunyaev, R.: Cosmic rays from microquasars: A narrow component to the CR spectrum?, Astron. Astrophys., 390, 751-766, 2002.

Jokipii, J. R.: Rate of energy gain and maximum energy in diffusive shock acceleration, Astrophys. J., 313, 842-846, 1987.

Kolmogorov, A. N.: Dissipation of Energy in the Locally Isotropic Turbulence, in: Turbulence and Stochastic Process: Kolmogorov's Ideas 50 Years On, 8 July 1991, translation: P. Roy. Soc. A-Math. Phy., 434, 9-13, 1991, Dokl. Akad. Nauk SSSR, 30, 301-305, 1941.
Krauss, M. I., Soderberg, A. M., Chomiuk, L., Zauderer, B. A., Brunthaler, A., Bietenholz, M. F., Chevalier, R. A., Fransson, C., and Rupen, M.: Expanded Very Large Array Observations of the Radio Evolution of SN 2011dh, Astrophys. J. Lett., 750, L40, doi:10.1088/2041-8205/750/2/L40, 2012.

Lagage, P. O. and Cesarsky, C. J.: The maximum energy of cosmic rays accelerated by supernova shocks, Astron. Astrophys., 125, 249-257, 1983.

Milisavljevic, D., Margutti, R., Soderberg, A. M., et al.: Multiwavelength Observations of Supernova 2011ei: Time-dependent Classification of Type IIb and Ib Supernovae and Implications for Their Progenitors, Astrophys. J., 767, 71, doi:10.1088/0004637X/767/1/71, 2013.

Soderberg, A. M., Kulkarni, S. R., Berger, E., Chevalier, R. A., Frail, D. A., Fox, D. B., and Walker, R. C. : The Radio and XRay-Luminous Type Ibc Supernova 2003L, Astrophys. J., 621, 908-920, 2005.

Soderberg, A. M., Chevalier, R. A., Kulkarni, S. R., and Frail, D. A.: The Radio and X-ray Luminous SN 2003bg and the Circumstellar Density Variations Around Radio Supernovae, Astrophys. J., 651, 1005-1018, 2006.

Soderberg, A. M., Brunthaler, A., Nakar, E., Chevalier, R. A., and Bietenholz, M. F.: Radio and X-ray Observations of the Type Ic SN 2007gr Reveal an Ordinary, Non-relativistic Explosion, Astrophys. J., 725, 922-930, 2010.

Tibolla, O., Vorster, M., Kaufmann, S., Ferreira, S., and Mannheim, K.: Are most of the VHE gamma-ray unidentified sources relic PWNe?, ICRC 2013, 4 pp., arXiv:1306.6833, 2013. 